\title{
Exploring the Potential and Challenges of VR Prototyping in Fashion Design
}

\author{
Jamil Joundi ${ }^{1,2}$, Peter Conradie ${ }^{1,2}$, Jelle Saldien ${ }^{1,2}$, Lieven De Marez ${ }^{1,3}$ \\ imec-MICT-UGent ${ }^{1}$ \\ Department of industrial Systems Engineering and Productdesign ${ }^{2}$ \\ Department of Communication Sciences ${ }^{3}$ \\ Ghent University, Ghent Belgium \\ \{jamil.joundi, peter.conradie, jelle.saldien, lieven.demarez\}@ugent.be
}

\begin{abstract}
This paper explores the possibilities of Virtual Reality (VR) as a tool for prototyping iterative design and development in the fashion industry. Subsequently, the system was evaluated by using two qualitative test protocols. Our results highlight how professional (fashion)designers view VR and what their expectations are.
\end{abstract}

\section{CCS CONCEPTS}

- Human-centered computing $\rightarrow$ Gestural input;

\section{KEYWORDS}

Computer aided design, virtual reality interaction, spatial user interface, 3D controller, fashion design, Belgium

\section{ACM Reference format:}

Jamil Joundi, Peter Conradie, Jelle Saldien and Lieven De Marez. 2018. Exploring the potential and challenges of VR prototyping in fashion design. In Proceedings of ACM SUI conference (SUI'18). ACM, Berlin, DE, Germany, 1 page.

https://doi.org/10.1145/3267782.3274768

\section{Developed system}

In this paper, we present a novel system for fashion designers to design clothes in VR. The setup was designed after performing a preliminary enquiry. In this enquiry, expert interviews in three different kinds of clothing companies $(\mathrm{A}, \mathrm{B}, \mathrm{C})$ were done where 10 managers, CTO's, lead fashion designers and one CEO were interviewed. The developed system consists of a new tangible controller with a pen that is attached to the standard HTC Vive controller to do precise actions. This pen tool was developed out of the users' need for more precision. In the VR software the shape of the clothes can be adjusted. A large 2D window was introduced in the VR scene, that shows the interface from the fashion design

Permission to make digital or hard copies of part or all of this work for personal or classroom use is granted without fee provided that copies are not made or distributed for profit or commercial advantage and that copies bear this notice and the full citation on the first page. Copyrights for third-party components of this work must be honored. For all other uses, contact the Owner/Author. SUI '18, October 13-14, 2018, Berlin, Germany

(C) 2018 Copyright is held by the owner/author(s)

ACM ISBN 978-1-4503-5708-1/18/10.

https://doi.org/10.1145/3267782.3274768 pc software CLO3D (fashion design software). The 3d-model was updated in real-time in the VR environment. For the interaction with the 2D window, a laser pointer pen was used. This interaction was intuitive. However, the precision should increase with a ruler and fixed work plane The adapted controllers allowed the user to use his hands to feel textile samples.

\section{Testing}

To explore the challenges and opportunities of VR prototyping, the developed system described above was subsequently evaluated within the three companies described (A, B, C). We performed a general impression evaluation (test 1) with 17 participants in total. The results highlight how professional fashion designers view VR and what their expectations are. We applied a think-aloud protocol [1] and sessions were also attended by persons not using the headset for providing additional feedback. Additionally, an in-depth user test was done by two fashion design interns in the companies A and B.

Visually, the designers would rather make a physical prototype because the visual quality was too low. Current limitations in usability and the new learning curve made it hard to win time in the development process. Currently, they use accurate software like illustrator or Lectra (2D) to materialize ideas.

From the user interviews and testing we learned that:

- real life scale VR helps fashion designers envision better how the clothes will look.

- $\quad$ having an interactive person (in different sizes) to fit on is much better than a mannequin.

At the end of the design process there is also an opportunity to use the developed system, when the design on the computer is finished, clothes can be rendered directly in the VR software. Decisions can then be made before having everything in visual physical prototypes. In this way, series can be judged, compared and communicated. In order for this system to be more implementable, further studies should be carried out to further define the role of digital tools in their design process. (for example, manual vs digital sketching, pattern refining, etc.)..It would help to carefully consider the workflow of the designers to adapt for VR tools.

\section{REFERENCES}

[1] Kjeldskov, J. and Skov, M.B. 2003. Creating Realistic Laboratory Settings Comparative Studies of Three Think-Aloud Usability Evaluations of a Mobile System. (2003). 